\title{
The effect of polarity on the lightning breakdown voltages of palm oil and coconut oil under a non-uniform field for transformers application
}

\begin{abstract}
This paper presents a study of the lightning breakdown voltages of Palm Oil (PO) and Coconut Oil (CO) under a non-uniform field with consideration on the polarity effect at various gap distances. All tests were carried based on a needle-sphere electrode configuration and various gap distances ranging from 2 to $25 \mathrm{~mm}$ under positive and negative voltage polarities. Three different testing techniques were used in this study including rising-voltage, up-and-down and multiple-voltage methods. The PO used in this study was Refined, Bleached and Deodorised Palm Oil (RBDPO) Olein. Three different samples of RBDPO and one sample of $\mathrm{CO}$ were tested. The Weibull distribution was used as a statistical approach to determine the withstand voltages of all samples at $1 \%$ and $50 \%$ probabilities for each type of oil. Under positive voltage polarity, it was found that the $50 \%$ breakdown voltages of RBDPO and CO were comparable with Mineral Oil (MO) whereby the highest percentage of difference among all gap distances was less than 15\%. RBDPO and CO have lower 50\% breakdown voltages than MO under a negative lightning impulse for which the highest percentage of difference can be up to $40 \%$.
\end{abstract}

Keyword: Palm oil; Coconut oil; Lightning breakdown voltage; Non-uniform field; Polarity effect; Transformers 